Article

\title{
Design of Broad Stopband Filters Based on Multilayer Electromagnetically Induced Transparency Metamaterial Structures
}

\author{
Ziyu Liu ${ }^{1}$, Limei Qi ${ }^{1, *(\mathbb{C})}$, Syed Mohsin Ali Shah ${ }^{1}$, Dandan Sun ${ }^{1}$ and Bin $\mathrm{Li}^{2, *}$ \\ 1 School of Electronic Engineering, Beijing University of Posts and Telecommunications, Beijing 100876, China; \\ lexi1022_lzy@bupt.cn (Z.L.); mohsin.shah32@gmail.com (S.M.A.S.); sdd661@bupt.edu.cn (D.S.) \\ 2 Beijing Research Center for Information Technology in Agriculture, Beijing 100097, China \\ * Correspondence: qilimei1204@163.com (L.Q.); Agrithz@163.com (B.L.); Tel.: +86-010-6228-1369 (L.Q.)
}

Received: 15 January 2019; Accepted: 11 March 2019; Published: 13 March 2019

\begin{abstract}
Broad stopband filters are proposed, based on multilayer electromagnetically induced transparency (EIT) metamaterial structures. The single EIT metamaterial consists of a U-shaped resonator and a strip on a polyimide substrate. The EIT-like spectral feature is firstly utilized to achieve stopband filters by properly coupling two layers of EIT structure. Influences of different rotation angles on the transmission properties of the two-layer EIT structure are investigated. It is found the wider low-transmission band can be obtained for the Transverse Magnetic (TM) polarization when the two EIT metal structures are vertical to each other. Furthermore, the bandwidth of the stopband can be controlled by increasing layers of the EIT structures with the proper architectural design. The design using a coupling effect of multi EIT-like resonances in the metamaterial would provide a new method for broad stopband filters in highly integrated optical circuits.
\end{abstract}

Keywords: stopband filter; electromagnetically induced transparency; multi-layer

\section{Introduction}

Electromagnetically induced transparency (EIT) is a spectrally narrow optical transmission window accompanied with extreme dispersion [1]. Within this spectral window, dramatically slowed down photons and orders of magnitude enhanced nonlinearities, can enable the manipulation of light at few-photon power levels [2-5]. Historically, EIT has been implemented in laser-driven atomic quantum systems [6]. However, limited material choices and stringent requirements to preserve the coherence of excitation pathways in atomic systems have significantly constrained the use of EIT effect. Recent studies have revealed that metamaterials can offer a versatile way to obtain the EIT-like optical responses [7,8]. Metamaterials are artificially engineered subwavelength materials which can be designed to display fascinating physical properties that cannot be easily accessed in natural materials $[9,10]$. For the EIT metamaterial structures, much of the research effort was so far focused on the EIT-like effect with a single resonance in single-layer metal-dielectric metamaterials [11,12] or in a cylindrical all-dielectric metamaterial with a high $Q$ of 1320 [13]. Once stacked in a multilayered structure, the presence of strong near-field coupling between the meta-atoms caused splitting or coupling of the EIT resonances, and lead to multispectral EIT-like behavior. Alp Artar et al. [1] introduced an approach enabling construction of a scalable metamaterial media to support multispectral plasmon-induced transparency. The composite multilayered media consists of coupled meta-atoms with radiant and subradiant hybridized plasmonic modes interacting through the structural asymmetry.

Zeng et al. [6] investigated the multiple electromagnetically induced transparencies (EIT)-like in graphene metamaterials consisting of a series of self-assembled graphene Fabry-Pérot (FP) 
cavities. The observed multiple EIT-like windows can be efficiently tuned in the mid-infrared regime by adjusting the Fermi level in graphene and the separations of FP cavities. Lu et al. [14] proposed that a metal-insulator-metal waveguide-resonator system performs a plasmonic analogue of electromagnetically induced transparency (EIT) in atomic systems, the plasmonic EIT-like response enables the realization of nanoscale bandpass filters with multiple channels by coupling with a series of side-coupled cavities and stub waveguides.

In this paper, broad stopband filters are proposed based on the multi EIT-like resonance in the multi-layer metamaterial structures. Each EIT metamaterial consists of a U-shaped resonator and a strip on a polyimide substrate which acts as bright and bright plasmonic modes coupling. It is found that the EIT-like spectral feature can be utilized to achieve broad stopband filters by proper design of different layers, and bandwidth of the stopband can be controlled by adding layers of the EIT structure with the proper architectural design. In fact, metamaterials with either electric or magnetic responses are inherent transmission band-stop filters, owing to their transmission dips. However, the bandwidth is always narrow due to the resonant nature of the response. Furthermore, to the best of our knowledge, there is little related work being carried out for the suppression of undesired responses or for the elimination of interfering signals in which wider forbidden bands are required [15]. Therefore, our design, by using a coupling effect of multi EIT-like resonances in the metamaterial would provide a new method for broad stopband filters in the highly integrated optical circuits.

\section{Design}

The side view of the unit cell of the single-layer EIT structure is shown in Figure 1a. It consists of two layers. The top layer is made of silver with the U-shaped resonator and a strip [16]. The second layer is selected as polyimide (PI) with the relative permittivity $\varepsilon_{d}=2.1$ and tangential $\operatorname{loss} \operatorname{tg} \delta=0.008$ [17]. The thicknesses of the metal and dielectric layers are $\mathrm{t} 1=20 \mathrm{~nm}$ and $\mathrm{t}=40 \mathrm{~nm}$, respectively. Permittivity of the silver is described by the Drude mode [18-20]:

$$
\varepsilon_{g}=1-\frac{\omega_{p}^{2}}{\omega^{2}+i \omega_{c} \omega}
$$

where, the plasma frequency is $\omega_{\mathrm{p}}=1.366 \times 10^{16} \mathrm{rad} / \mathrm{s}$ and the collision frequency is $\omega_{\mathrm{c}}=3.07 \times 10^{13} \mathrm{rad} / \mathrm{s}[16,18]$. The dimensions of the other parameters are $\mathrm{P}=400 \mathrm{~nm}, \mathrm{~L} 1=130 \mathrm{~nm}$, $\mathrm{L} 2=55 \mathrm{~nm}, \mathrm{~L} 3=130 \mathrm{~nm}, \mathrm{~L} 4=140 \mathrm{~nm}, \mathrm{~d}=35 \mathrm{~nm}, \mathrm{~g}=45 \mathrm{~nm}, \mathrm{~h} 1=80 \mathrm{~nm}$, and h2 = h3 = $135 \mathrm{~nm}$.

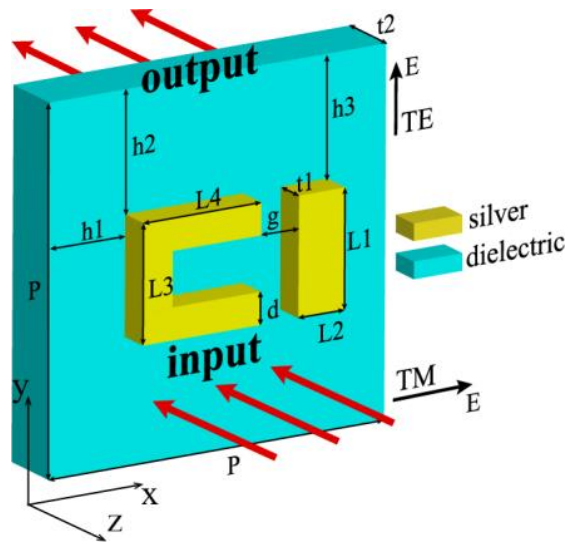

Figure 1. Side view of the unit cell of the single-layer electromagnetically induced transparency (EIT) structure. The dimensions of parameters are P $=400 \mathrm{~nm}, \mathrm{~L} 1=130 \mathrm{~nm}, \mathrm{~L} 2=55 \mathrm{~nm}, \mathrm{~L} 3=130 \mathrm{~nm}$, $\mathrm{L} 4=140 \mathrm{~nm}, \mathrm{~d}=35 \mathrm{~nm}, \mathrm{~g}=45 \mathrm{~nm}, \mathrm{~h} 1=80 \mathrm{~nm}$, and h2 = h3 = $135 \mathrm{~nm}$.

In the simulation, commercial software (CST Microwave Studio) is used, and the frequency domain solver is selected to obtain the transmission $S_{21}$ and the reflection $S_{11}$. We use periodic 
boundary conditions along $x$ and $y$ axes for the unit cell. For a plane wave which would be normally incident into the surface of the EIT structure from the $-\mathrm{z}$ axis, the input and output ports are denoted by the red arrows in Figure 1a. The distances between the ports and the surfaces of the EIT structure are assigned automatically by the CST software as the open (add space) boundary conditions are used along the $\mathrm{z}$-axis for the unit cell. The electromagnetic wave can be divided into the Transverse Electric (TE) polarization where the electric field is parallel to the $y$-axis and the TM polarization where the electric field is parallel to the $\mathrm{x}$-axis (denoted in Figure 1).

\section{Simulations and Results}

Figure $2 \mathrm{a}$ shows the transmission and the reflection spectra of the single-layered EIT structure. The top and the bottom layers are corresponding to the TE and TM polarizations, respectively. The solid and dotted lines denote the transmission and reflection curves, respectively. For the TE polarization, the transmission shows the EIT effect at the peak $\mathrm{f} 2=420 \mathrm{THz}$ accompanied by two dips at $\mathrm{f} 1=396$ $\mathrm{THz}$ and $\mathrm{f} 3=436 \mathrm{THz}$, respectively, while for the TM polarization, there is only one dip at $\mathrm{f} 4=353 \mathrm{THz}$. Generally, the EIT phenomenon observed in metamaterials can be realized by the bright-bright mode coupling [21] or the bright-dark mode coupling [22]. Classical analogy of EIT effect in metamaterials was initially observed in arrays of asymmetrically split rings (ASRs). The asymmetric coupling between the two bright modes can excite a high-Q mode formed by counter-propagating currents, i.e., a trapped mode resonance [23]. To further understand the physical mechanism of the single-layer EIT structure in Figure 1, Figure 2b shows the electric field distributions of the two polarizations. For the three frequencies on the top layer of the TE polarization, the electric field in the strip is strongly excited at the first dip f1 = $396 \mathrm{THz}$, while the U-shaped resonator is weakly excited, which means the strip works as a bright mode at $\mathrm{f} 1=396 \mathrm{THz}$. For the second $\operatorname{dip}$ at $\mathrm{f} 3=436 \mathrm{THz}$, the left part of the U-shaped structure couples strongly to the incident light, while the two parts on the top and bottom sides have the opposite electric field distributions, which supports the bright mode as the strip at the first dip [24]. At the resonance peak of $\mathrm{f} 2=420 \mathrm{THz}$, both the $\mathrm{U}$ shape and the strip are excited simultaneously due to the resonance detuning, and this results in the reverse induced current or electric field distributions for the strip and the left side of the $U$ shape, which corresponds to the characteristics of the trapped mode resonance $[16,23,25,26]$.

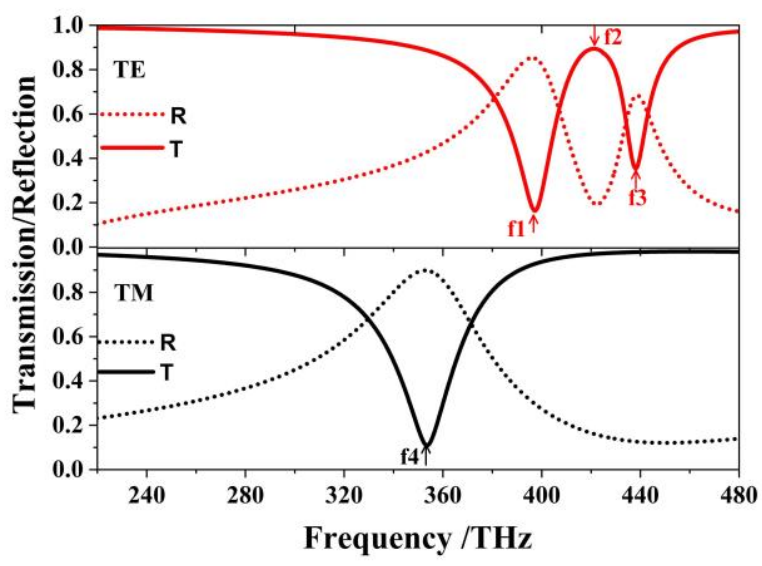

(a)

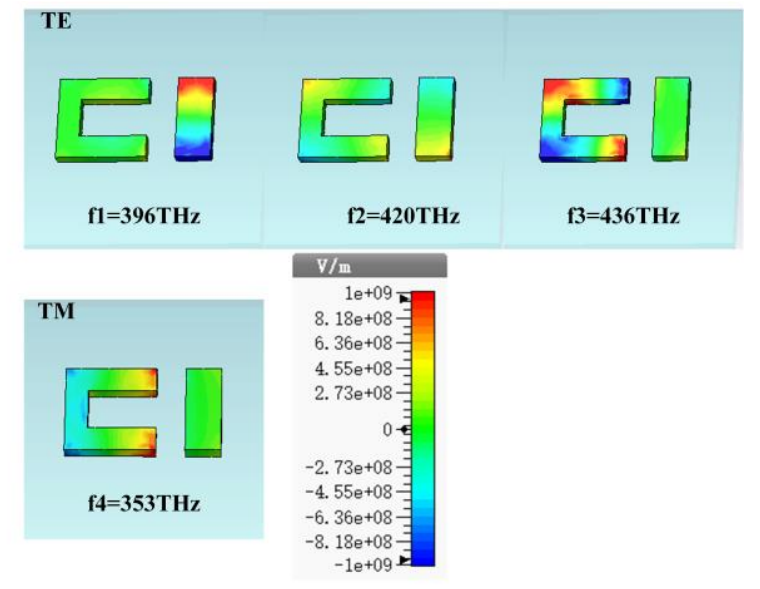

(b)

Figure 2. (a) The transmission and reflection spectra of the single-layer EIT structure at the normal incidence. (b) Electric field distributions of the resonant frequencies at $\mathrm{f} 1=396 \mathrm{THz}, \mathrm{f} 2=420 \mathrm{THz}$ and $\mathrm{f} 3=436 \mathrm{THz}$ for the Transverse Electric (TE) polarization, and $\mathrm{f} 4=353 \mathrm{THz}$ for the Transverse Magnetic (TM) polarization. 
As a result, a transparency window appears in the transmission spectra. For the TM polarization, there is only one dip at $\mathrm{f} 4=353 \mathrm{THz}$. Based on the distribution of the electric field on the bottom layer, the radiation of the U-shaped resonator is apparently working as a bright mode $[21,27,28]$.

Figure 3a shows the side view of a two-layer EIT structure by rotating the metal structure of the second layer with a clockwise angle $\phi$ along the $z$-axis $[15,29,30]$. The distance between the two layers is $\mathrm{S}$. For the two-layer EIT structure with $\mathrm{S}=0$, Figure $3 \mathrm{~b}, \mathrm{c}$ gives the color map of transmission curves as $\phi$ varying from $0^{\circ}$ to $180^{\circ}$ for the TE and the TM polarizations, respectively. The red and blue color areas correspond to the high-transmission and low-transmission regions, respectively. It is found that there are several regions of low-transmission bands for the two-layer EIT structure. For the TM polarization, the widest low-transmission band varies from $308 \mathrm{THz}$ to $387 \mathrm{THz}$ at $\phi=90^{\circ}$, which means the broadest stopband filter could be achieved by rotating the angle with $\phi=90^{\circ}$ for the two single-layer EIT structures. For the same EIT structure with $\phi=90^{\circ}$, Figure $4 \mathrm{a}$, b shows the color map of transmission curves as the distance $S$ varying from $0 \mathrm{~nm}$ to $20 \mathrm{~nm}$, for (a) the TE polarization, and (b) the TM polarization. For the TE polarization, there are always three low-transmission regions and this cannot form the broad stopband properties as $S$ increases from $0 \mathrm{~nm}$ to $20 \mathrm{~nm}$. As for the TM polarization, there is, firstly, a broadband low-transmission region when $S$ is smaller than $2 \mathrm{~nm}$, but then it splits into three linear curves, and the distances between them tend to enlarge as $S$ increases. Therefore, increasing the distance between two layers of EIT structure will not obtain a wide stopband filter.

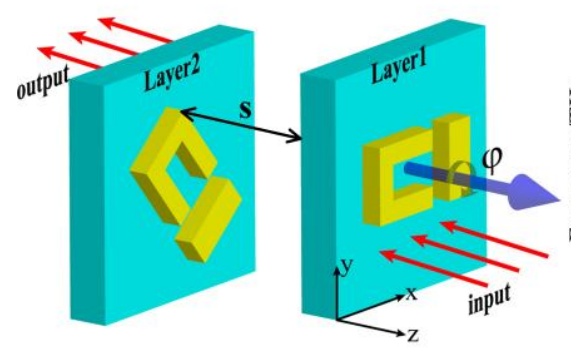

(a)

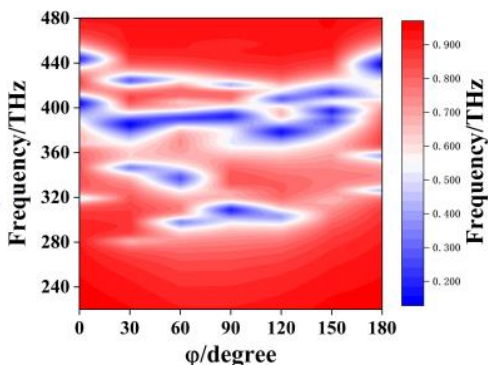

(b)TE

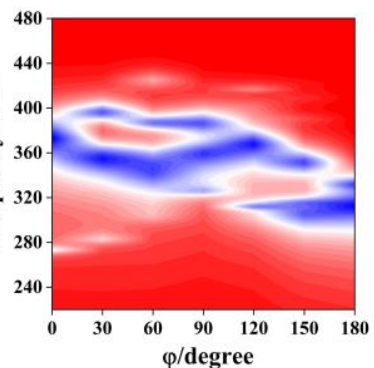

(c) TM

Figure 3. (a) The two-layer EIT structure by rotating the metal structure of the second layer with a clockwise angle $\phi$ along the z-axis. The color map of transmission curves of the two-layer structure with $\phi$ varying from $0^{\circ}$ to $180^{\circ}$ for (b) the TE polarization and (c) the TM polarization.

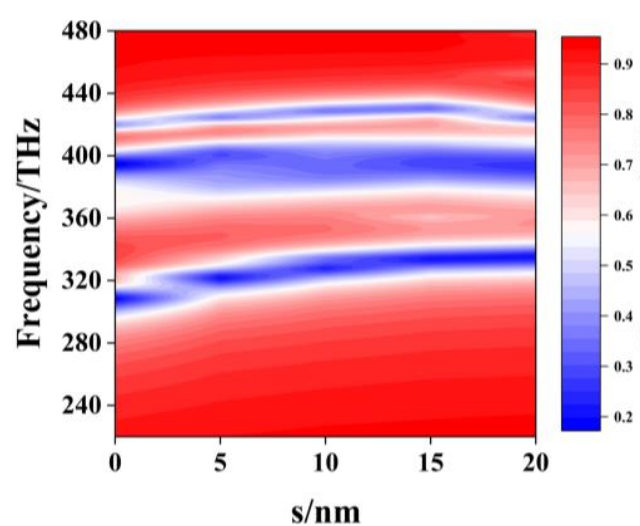

(a) TE

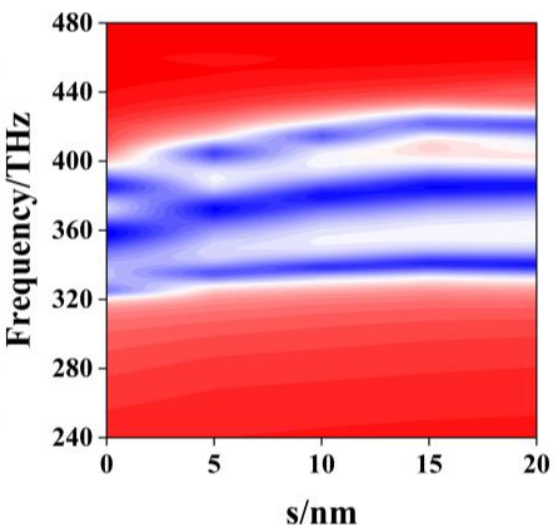

(b) TM

Figure 4. The color map of transmission curves of the two-layer structure with the distance $S$ varying from $0 \mathrm{~nm}$ to $20 \mathrm{~nm}$ at $\phi=90^{\circ}$ for (a) the TE polarization and (b) the TM polarization.

Secondly, it is very difficult to keep a small gap between two layers both in the fabrication and experiment. In the following, we only consider the multilayer structures with the distance $S=0$. 
Figure $5 \mathrm{~b}$ shows the transmission spectrum of the TE and TM polarizations of the stopband properties of the two-layer EIT structure with $90^{\circ}$ rotation and distance $\mathrm{S}=0$ (in Figure 5a). Compared with the single-layer EIT structure in Figure 2, three transmission dips appear for each polarization in the two-layer structure due to the hybridization effect and superposition principle between the two layers. Electric field distributions of the resonances are illustrated in Figure 5c. The top layer shows results of the three dips at $\mathrm{f} 1=308 \mathrm{THz}, \mathrm{f} 2=393 \mathrm{THz}$ and $\mathrm{f} 3=419 \mathrm{THz}$ for the TE polarization, the middle layer shows these of the two peaks at $\mathrm{f} 4=338 \mathrm{THz}$ and $\mathrm{f} 5=410 \mathrm{THz}$ for the TE polarization. The bottom layer represents the results at $\mathrm{f} 6=326 \mathrm{THz}, \mathrm{f} 7=358 \mathrm{THz}$ and $\mathrm{f} 8=387$ $\mathrm{THz}$ for the TM polarization. For the TE polarization, the U-shaped resonator of the second layer at $\mathrm{f} 1=308 \mathrm{THz}$ is strongly excited, which has the same field distribution as the first layer under TM polarization. At f2 $=393 \mathrm{THz}$, the strip of the first layer and U-shaped resonator of the second layer are excited simultaneously, but the strip has the stronger field than the U-shaped structure. The field distribution of the first layer is nearly the same as that of the single layer EIT structure at 396THz for TE polarization, as is shown in Figure $2 \mathrm{~b}$. At $\mathrm{f} 3=419 \mathrm{THz}$, the U-shaped resonator of the first layer is excited and has the similar characteristic of U-shaped resonator of single-layered EIT structure at $436 \mathrm{THz}$.

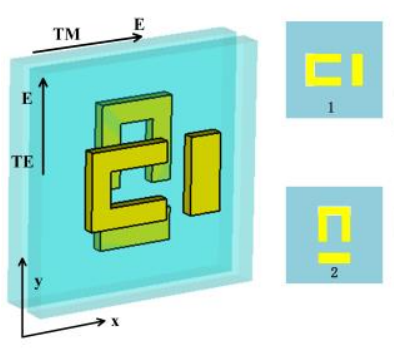

(a)

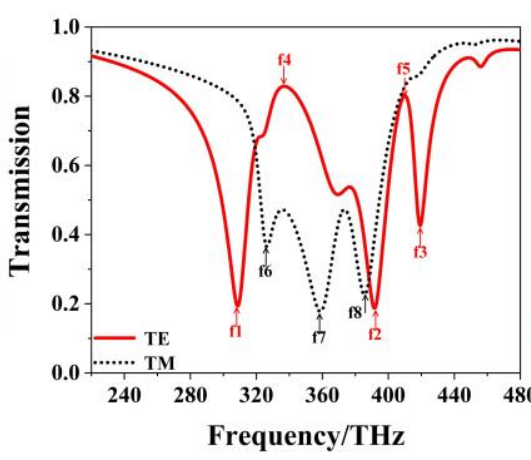

(b)

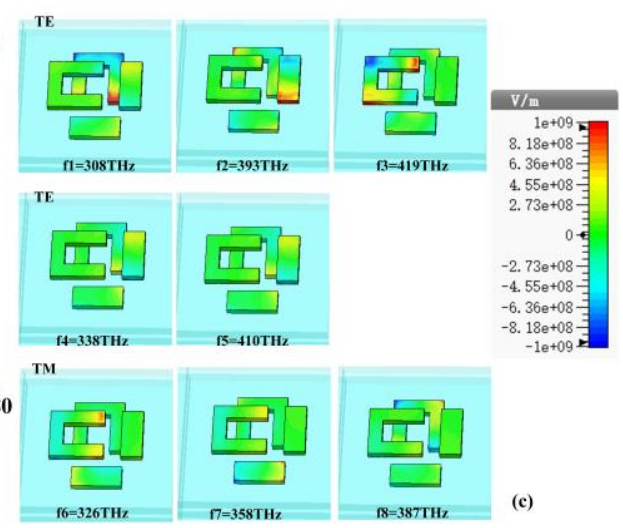

Figure 5. (a) Side view of the two-layer EIT structure, where the second EIT structure is formed by rotating the metal structure with $90^{\circ}$ from the first structure, (b) transmission spectrum of the TE (solid line) and the TM polarizations (dotted line). (c) Electric field distributions of eight resonant frequencies for the TE (top layer) and TM polarizations.

For the transmission peaks at $\mathrm{f} 4=338 \mathrm{THz}$ and $\mathrm{f} 5=410 \mathrm{THz}$, similar weak electric field distributions can be seen on the two layers, where the strip on the first layer and the right part of U-shaped resonator on the second layer have inverse parallel electric field distributions like the characteristics of electromagnetically-trapped mode of two resonators in a single-layer EIT structure [16,25]. Therefore, the transmission properties of the two-layer EIT structures for the TE polarization are caused by superposition of the two layers of EIT structures. As for the TM polarization, it is observed that the U-shaped resonator on the first layer is excited at $\mathrm{f} 6=326 \mathrm{THz}$, while the others are excited weakly. At $\mathrm{f} 7=358 \mathrm{THz}$, both the U-shaped resonator on the first layer and the strip on the second layer are excited by an incident wave. However, the field in the strip of the second layer is stronger and contributes more to the resonant frequency $\mathrm{f} 7=358 \mathrm{THz}$. At $\mathrm{f} 8=387 \mathrm{THz}$, the field concentrates on the U-shaped resonator of the second layer, which has the similar field distribution of the first layer under the TE polarization as is shown in Figure 2b. Based on the field distributions at the three dips, the transmission of the TM polarization is also the superposition of each layer.

Based on the two-layer EIT structure, a wide low-transmission band appears due to the EIT superposition, which can be designed as a wide stopband filter. Figure 6 shows the transmission spectra of three types of EIT structures with single layer, two layers and three layers for (a) TE polarization and (b) TM polarization. The dot, dot-dash and solid lines denote the results of the single-layer, two-layer and three-layer structures, respectively. Both for the two polarizations, we can 
see that as the number of the EIT layers increases, more dips will appear in the stop band, which makes a wider stopband in the transmission spectrum. By focusing on the dips of three different layers, it is easy to see that the dips of the three-layer structure can be seen as the superposition of the single-layer and two-layer EIT structures both for the two polarizations, and the broadest stopband filter is obtained for three-layer structure with the TM polarization.

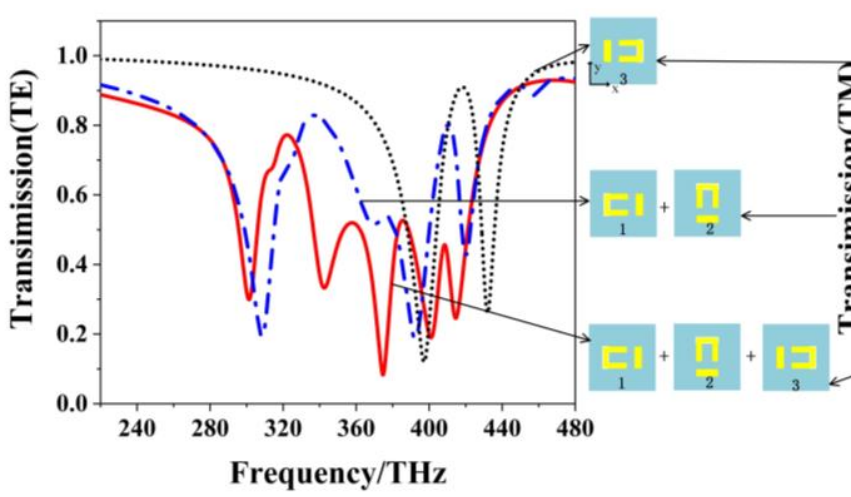

(a)TE

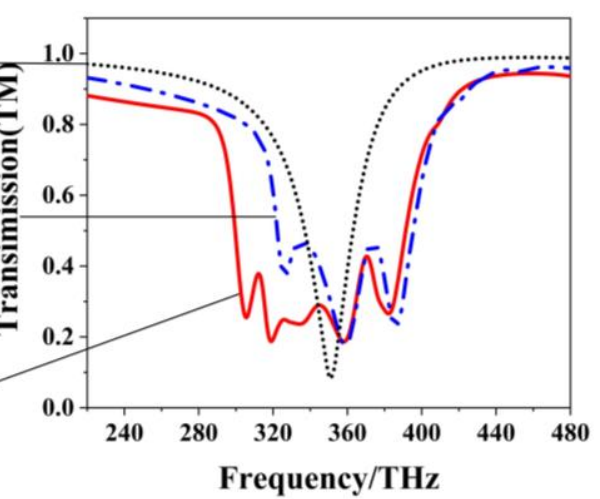

(b) TM

Figure 6. The transmission spectra for three types of EIT structure with single layer, two layers and three layers for (a) TE polarization, and (b) TM polarization.

In the insert figures in Figure 6, the rotating angle $\phi$ of each two close layers is $90^{\circ}$ for the three-layer EIT structure. To generalize, Figure 7 shows the color map of transmission curves for the three-layer structure with $\phi$ of each two close layers varying from $0^{\circ}$ to $180^{\circ}$. For the TE polarization, there are several regions of narrow low-transmission bands, which is difficult to form a broad stopband filter. For the TM polarization, it is easy to form the broad low-transmission region as $\phi$ locating between $70^{\circ}$ to $140^{\circ}$. However, the widest low-transmission band still appears at $\phi=90^{\circ}$ for the three-layer structure.

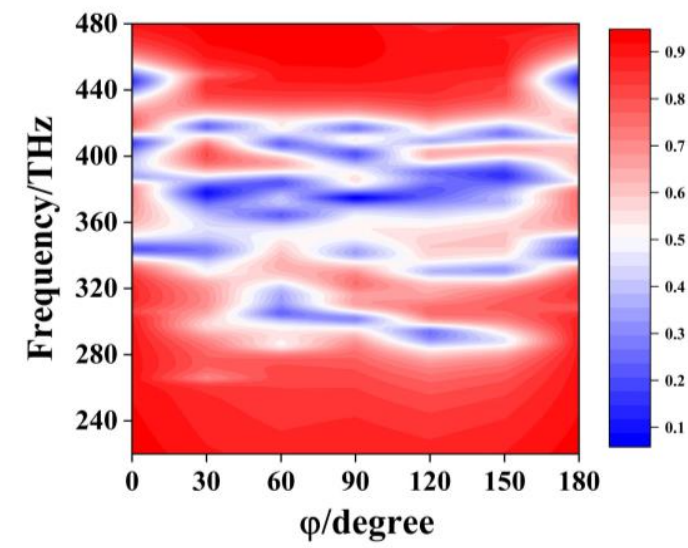

(a)TE

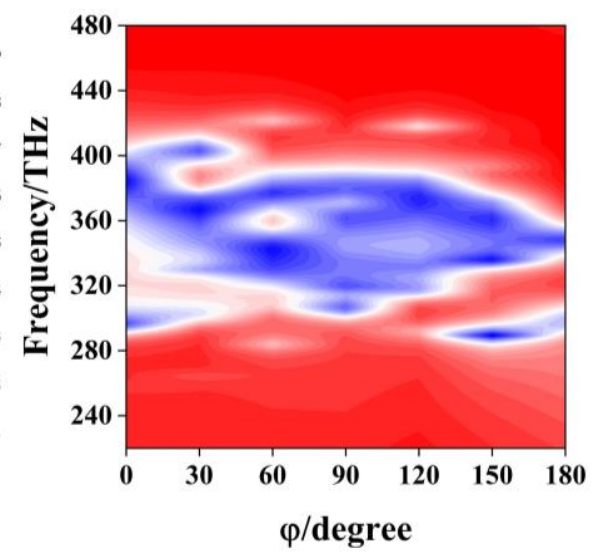

(b) TM

Figure 7. The color map of transmission curves for the three-layer structure with $\phi$ of each two close layers varying from $0^{\circ}$ to $180^{\circ}$ for (a) the TE polarization and (b) the TM polarization.

By introducing the 4th-layer metamaterial into the three-layer EIT structure, a four-layer EIT structure is constructed in Figure 8. For the four-layer EIT structure, the second, third and the fourth layers are the results of $90^{\circ}, 180^{\circ}, 270^{\circ}$ rotating of the first layer. The dotted, dot-dash and solid lines denote the results of the single-layer, three-layer and four-layer structures, respectively. For the TE polarization, compared with the three-layer structure, bandwidth of the four-layer structure changes 
little, but better stopband properties are obtained with a sharp out-of-band rejection on the left side. For the TM polarization, it is also seen that the widest stopband is obtained for the four-layer structure.

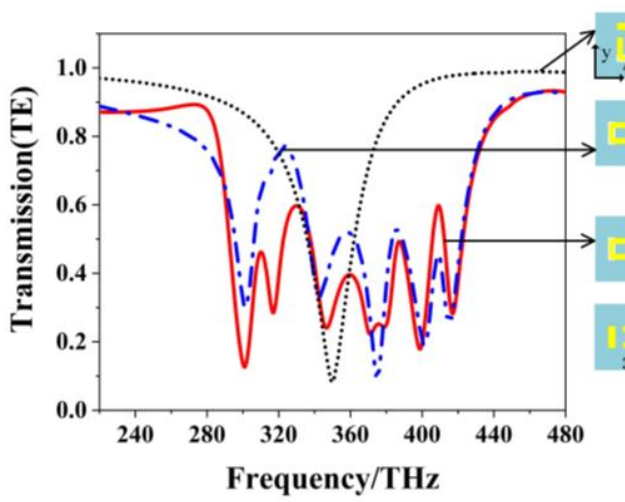

(a) $\mathrm{TE}$

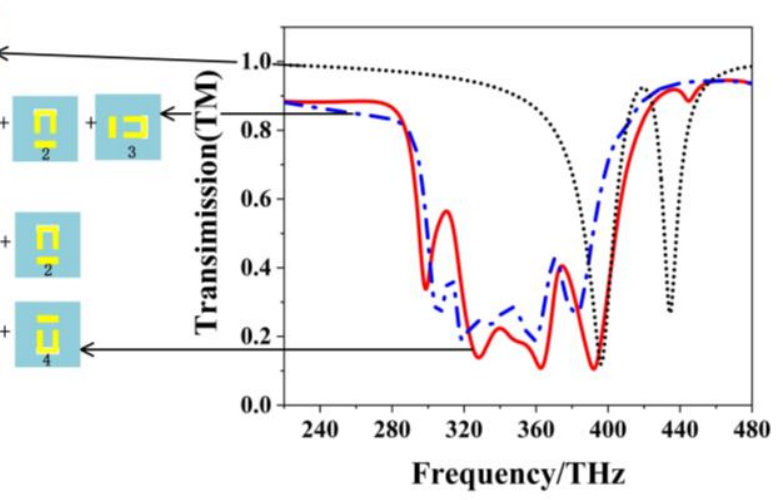

(b) $\mathrm{TM}$

Figure 8. The transmission spectra of three types of EIT structure with single layer, three layers and four layers for (a) TE polarization, and (b) TM polarization.

A 5th-layer metamaterial is introduced into the four-layer EIT structure to get the wider stopband filter in Figure 8, forming a five-layer EIT structure. The last layer is the $360^{\circ}$ rotation of the first layer, which has the same direction as the first one. Figure 9 shows the transmission of the five-layer structure. The solid and dot-dashed lines denote the result of the TE and TM polarizations, respectively. It is seen that a wider band-stop filter is obtained due to the superposition of multi-EIT response. For the TM polarization, the bandwidth is wider with low transmission that is lower than 0.3 in the stopband, while a transmission peak of 0.68 appears at $322 \mathrm{THz}$ for TE polarization.

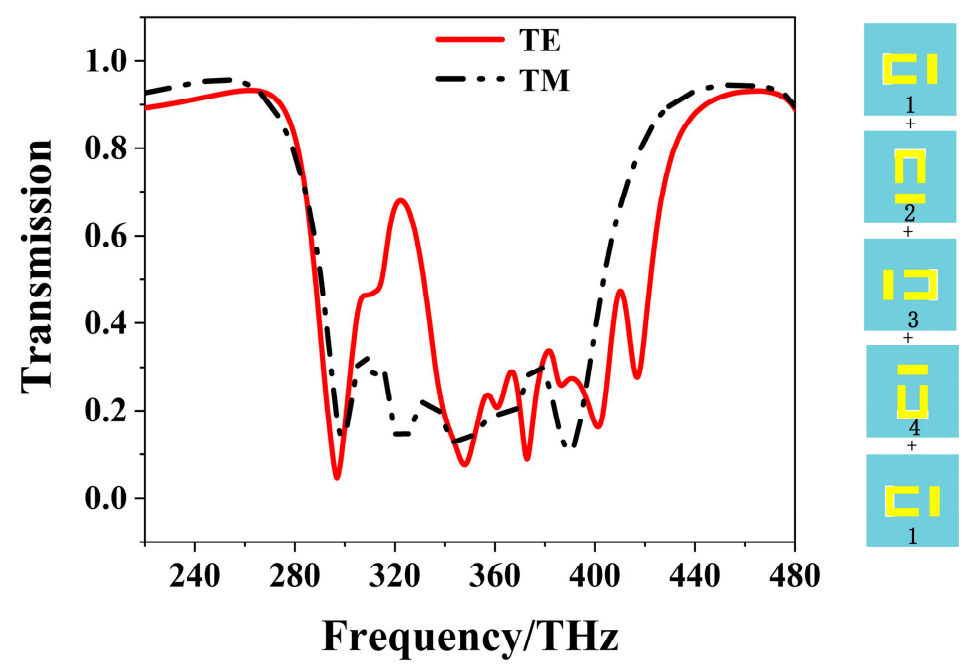

Figure 9. The transmission spectra of five-layer EIT structure for TE polarization and TM polarization.

According to the discussions above, the widest stopband characteristics can be obtained for the five-layer structure at the normal incidence. For the same five-layer structure in Figure 9, Figure 10 shows the color map of the transmission spectra at obliquely incident angles varying from $0^{\circ}$ to $80^{\circ}$. 


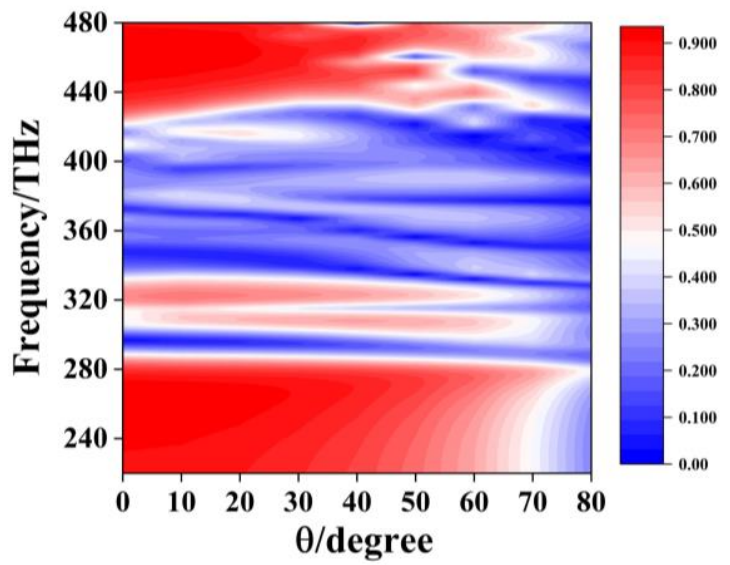

(a) TE

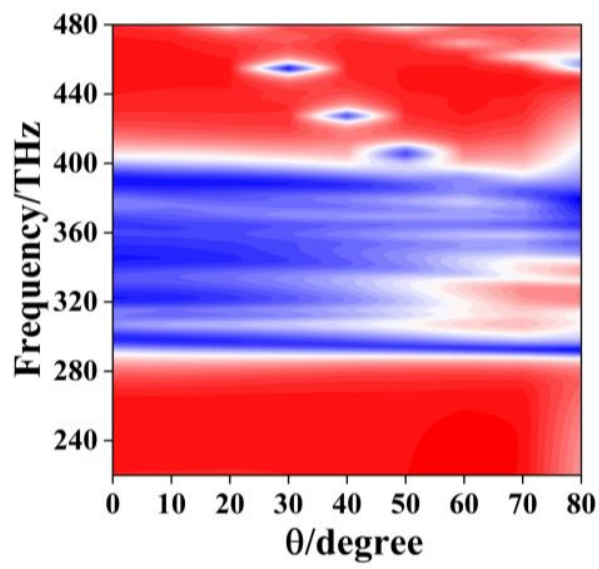

(b) TM

Figure 10. Color map of the transmission spectra for (a) the TE and (b) the TM polarizations for the five-layer system at obliquely incident angle.

We can see that wide stopband filters can be obtained for the two polarizations in the frequency ranges of $220 \mathrm{THz}$ to $480 \mathrm{THz}$. However, a narrow stopband always exists for the TE polarization. For the TM polarization, the wider stopband filters always appear at the incident angles below $50^{\circ}$.

For the five-layer structure, the whole thickness is only $300 \mathrm{~nm}$, therefore a substrate is needed for this ultrathin device in practice. Figure 11 shows the transmission spectra of the five-layer system on the substrate of polyimide (PI) and $\mathrm{SiO}_{2}$ for the $\mathrm{TM}$ polarization at normal incidence. For the $\mathrm{SiO}_{2}$, the relative permittivity $\varepsilon_{\mathrm{d}}=4.41$ and tangential loss $\operatorname{tg} \delta=0.0004$ [31] are used in the simulation. The solid, dotted and dot-dashed lines correspond to the results of thickness of substrate PI $h=0$, 30 and $70 \mathrm{um}$, and it is also seen that the property of broad stopband still exists except for a little frequency shift and increase of magnitude in the stop band for the five-layer structure. If $\mathrm{SiO}_{2} \mathrm{with}$ $\mathrm{h}=10 \mathrm{um}$ is used as the substrate, the stopband on the left side shifts to the lower frequency because the increase of the dielectric constant of the whole structure will lead to the decrease of the frequency of the modes according to the variational principles [32,33]. To fabricate the multilayer metamaterial structure, we can firstly get the single-layer EIT structure on the substrate by using electron-beam lithography [24]. As the resolution of the electron-beam lithography system can be controlled within $10 \mathrm{~nm}$ [34], the dimensions of the EIT structure given in Figure 1 can be realized in experiment. The multilayer structure can finally be formed by repeating the procedure of single-layer fabrication several times [35].

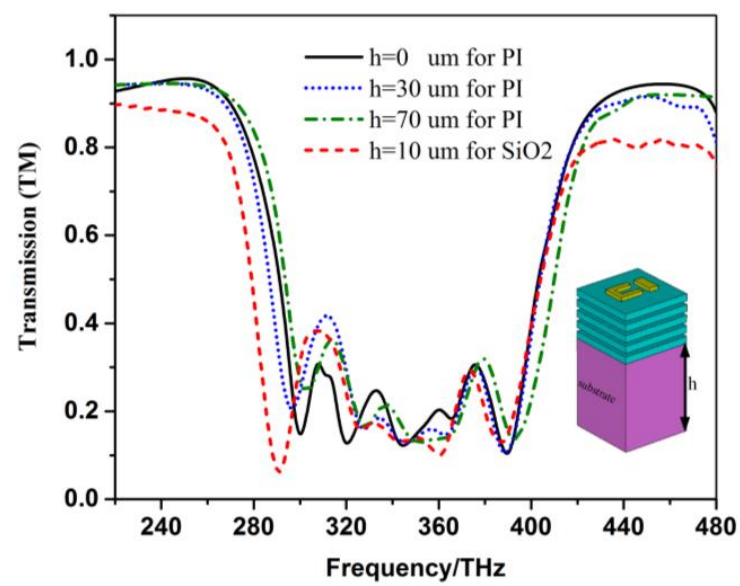

Figure 11. Transmission spectra of the five-layer system on the substrate of PI and $\mathrm{SiO} 2$ for the TM polarization at normal incidence. 


\section{Conclusions}

Broad stopband filters are firstly proposed based on the multi EIT-like resonance in the multi-layer metamaterial structures. Firstly, the physical mechanism of the single-layer EIT structure is given both for the TE and TM polarizations. Then, by investigating the rotation angle on the transmission properties of the two-layer EIT structure, we found that the wider low-transmission band can be obtained for the TM polarization when the two metal resonant structures are vertical to each other. Furthermore, it is found that the bandwidth of the stopband can be controlled by increasing layers of the EIT structure with the proper architectural design, and the wider stopband filter is obtained due to the superposition of multi-EIT response. By comparing the properties of the two-layer, three-layer, four-layer and five-layer EIT structures, the widest stopband filter is obtained for the five-layer structure. For the same five-layer structure, the color map of the transmission spectra at obliquely incident angles varying from $0^{\circ}$ to $80^{\circ}$, is also given, and a wider band always appears for the incident angles below $50^{\circ}$ for the TM polarization. As different substrates are introduced for the five-layer EIT structure, the broad stopband still exists except for some frequency shift and any increase of the magnitude in the stop band. The proposed broad stopband filters are promising for suppressing undesired responses or eliminating interfering signals within a wide frequency range. Furthermore, while we focused only on a specific multilayer EIT-like metamaterial in the optical frequency, the general principle can be also extended to the longer wavelengths such as terahertz and microwave frequencies, where it is easier to realize the multilayer structure in the manufacturing process.

Author Contributions: Conceptualization, Z.L. and L.Q.; simulation, Z.L.; writing-original draft preparation, Z.L.; writing-review and editing, D.S., L.Q., S.M.A.S. and B.L.; supervision, L.Q.

Funding: This research was funded by the National Natural Science Foundation of China (grant number 61875017 and 61107030), and by The Science and Technology Innovation Special Construction Funded Program of Beijing Academy of Agriculture and Forestry Sciences (grant number KJCX20180119).

Acknowledgments: This work is supported by the Fundamental Research Funds for the Central Universities of China and by the Opening Foundation of the State Key Laboratory of Millimeter Waves.

Conflicts of Interest: The authors declare no conflict of interest.

\section{References}

1. Artar, A.; Yanik, A.A.; Altug, H. Multispectral Plasmon Induced Transparency in Coupled Meta-Atoms. Nano Lett. 2011, 11, 1685-1689. [CrossRef]

2. Marangos, J.P. Electromagnetically induced transparency. J. Mod. Opt. 1998, 45, 471-503. [CrossRef]

3. Zhang, K.; Wang, C.; Qin, L.; Peng, R.W.; Xu, D.H.; Xiong, X.; Wang, M. Dual-mode electromagnetically induced transparency and slow light in a terahertz metamaterial. Opt. Lett. 2014, 39, 3539-3542. [CrossRef]

4. Harris, S.; Hau, L.V. Nonlinear optics at low light levels. Phys. Rev. Lett. 1999, 82, 4611-4614. [CrossRef]

5. Zhu, L.; Meng, F.Y.; Fu, J.H.; Wu, Q.; Hua, J. Multi-band slow light metamaterial. Opt. Express 2018, 20, 4494-4502. [CrossRef]

6. Zeng, C.; Cui, Y.D.; Liu, X.M. Tunable multiple phase-coupled plasmon induced transparencies in graphene metamaterials. Opt. Express 2015, 23, 545-551. [CrossRef]

7. Papasimakis, N.; Zheludev, N.I. Metamaterial-induced transparency: Sharp Fano Resonances and Slow light. Opt. Photonics News. 2009, 20, 22-27. [CrossRef]

8. Zhang, S.; Genov, D.A.; Wang, Y.; Liu, M.; Zhang, X. Plasmon-induced transparency in metamaterials. Phys. Rev. Lett. 2008, 101, 047401. [CrossRef]

9. Smith, D.R.; Pendry, J.B.; Wiltshire, M.C.K. Metamaterials and Negative Refractive Index. Science 2004, 305, 788-792. [CrossRef]

10. Zheludev, N.I. The Road Ahead for Metamaterials. Science 2010, 328, 582-583. [CrossRef]

11. Nakanishi, T.; Kitano, M. Storage and retrieval of electromagnetic waves using electromagnetically induced transparency in a nonlinear metamaterial. Appl. Phys. Lett. 2018, 112, 201905. [CrossRef] 
12. Ling, Y.H.; Huang, L.R.; Hong, W.; Liu, T.J.; Luan, J.; Liu, W.B.; Lai, J.J.; Li, H.P. Polarization-controlled dynamically switchable plasmon-induced transparency in plasmonic metamaterial. Nanoscale 2018, 10, 19517-19523. [CrossRef]

13. Ospanova, A.K.; Karabchevsky, A.; Basharin, A.A. Metamaterial engineered transparency due to the nullifying of multipole moments. Opt. Lett. 2018, 43, 503-506. [CrossRef]

14. Lu, H.; Liu, X.M.; Wang, G.X.; Mao, D. Tunable high-channel-count bandpass plasmonic filters based on an analogue of electromagnetically induced transparency. Nanotechnology 2012, 23, 444003. [CrossRef]

15. Li, X.; Yang, L.Y.; Hu, C.G.; Luo, X.G.; Hong, M.H. Tunable bandwidth of band-stop filter by metamaterial cell coupling in optical frequency. Opt. Express 2011, 19, 5283-5289. [CrossRef]

16. Liu, R.; Na, B.; Shi, J.H.; Wang, Z.P. Multiple transmission windows in a bilayered metamaterial based on twisted asymmetrically split rings. In Proceedings of the SPIE Nanophotonics and Micro/Nano Optics, 85640W, Beijing, China, 20 November 2012. [CrossRef]

17. Shao, J.; Li, J.; Li, J.Q.; Wang, Y.K.; Dong, Z.G.; Lu, W.B.; Zhai, Y. The metamaterial analogue of electromagnetically induced transparency by dual-mode excitation of a symmetric resonator. Chin. Phys. B 2013, 22, 107804. [CrossRef]

18. Jin, X.R.; Zhang, Y.Q.; Zhang, S.; Lee, Y.P.; Rhee, J.Y. Polarization-independent electromagnetically induced transparency-likeeffects in stacked metamaterials based on Fabry-Pérot resonance. J. Opt. 2013, 15, 125104. [CrossRef]

19. Liu, N.; Langguth, L.; Weiss, T.; Kästel, J.; Fleischhauer, M.; Pfau, T.; Giessen, H. Plasmonic analogue of electromagnetically induced transparency at the Drude damping limit. Nat. Mater. 2009, 8, 758-762. [CrossRef]

20. Ordal, M.A.; Long, L.L.; Bell, R.J.; Bell, S.E.; Bell, R.R.; Alexander, R.W.; Ward, C.A., Jr. Optical properties of the metals $\mathrm{Al}, \mathrm{Co}, \mathrm{Cu}, \mathrm{Au}, \mathrm{Fe}, \mathrm{Pb}, \mathrm{Ni}, \mathrm{Pd}, \mathrm{Pt}, \mathrm{Ag}, \mathrm{Ti}$, and $\mathrm{W}$ in the infrared and far infrared. Appl. Opt. 1983, 22, 1099-1119. [CrossRef]

21. Jin, X.R.; Park, J.W.; Zheng, H.Y.; Lee, S.J.; Lee, Y.P.; Rhee, J.Y.; Kim, K.W.; Cheong, H.S.; Jang, W.H. Highly-dispersive transparency at optical frequencies in planar metamaterials based on two-bright-mode coupling. Opt. Express 2011, 19, 21652-21657. [CrossRef]

22. Zhang, L.; Tassin, P.; Koschny, T.; Kurter, C.; Anlage, S.M.; Soukoulis, C.M. Large group delay in a microwave metamaterial analog of electromagnetically induced transparency. Appl. Phys. Lett. 2010, 97, 241904. [CrossRef]

23. Fedotov, V.A.; Rose, M.; Prosvirnin, S.L.; Papasimakis, N.; Zheludev, N.I. Sharp trapped-mode resonances in planar metamaterials with a broken structural symmetry. Phys. Rev. Lett. 2007, 99, 147401. [CrossRef]

24. Zhang, J.J.; Xiao, S.S.; Jeppesen, C.; Kristensen, A.; Mortensen, N.A. Electromagnetically induced transparency in metamaterials at near-infrared frequency. Opt. Express 2010, 18, 17187-17192. [CrossRef]

25. Plum, E.; Fedotov, V.A.; Kuo, P.; Tsai, D.P.; Zheludev, N.I. Towards the lasing spaser: Controlling metamaterial optical response with semiconductor quantum dots. Opt. Express 2009, 17, 8548-8551. [CrossRef]

26. Liu, T.T.; Wang, H.X.; Liu, Y.; Xiao, L.S.; Zhou, C.B.; Xu, C.; Xiao, S.Y. Dynamically tunable electromagnetically induced transparency in a terahertz hybrid metamaterial. Phys. E Low-Dimens. Syst. Nanostruct. 2018, 104, 229-232. [CrossRef]

27. Zhang, H.Y.; Cao, Y.Y.; Liu, Y.Z.; Liu, Y.; Zhang, Y.P. A novel graphene metamaterial design for tunable terahertz plasmon induced transparency by two bright mode coupling. Opt. Commun. 2017, 391, 9-15. [CrossRef]

28. Yu, W.; Meng, H.Y.; Chen, Z.J.; Li, X.P.; Zhang, X.; Wang, F.Q.; Wei, Z.C.; Tan, C.H.; Huang, X.G.; Li, S.T. The bright-bright and bright-dark mode coupling-based planar metamaterial for plasmonic EIT-like effect. Opt. Commun. 2018, 414, 29-33. [CrossRef]

29. Shi, J.H.; Ma, H.F.; Jiang, W.X.; Cui, T.J. Multiband stereometamaterial-based polarization spectral filter. Phys. Rev. B 2012, 86, 035103. [CrossRef]

30. Liu, R.; Na, B.; Xu, Y.Q.; Zhu, Z.; Wang, Y.K.; Ma, H.F.; Cui, T.J. Engineering electromagnetic responses of bilayered metamaterials based on Fano resonances. Appl. Phys. Lett. 2013, 103, 071906.

31. Lan, F.; Yang, Z.Q.; Qi, L.M.; Gao, X.; Shi, Z.J. Terahertz dual-resonance bandpass filter using bilayer reformative complementary metamaterial structures. Opt. Lett. 2014, 39, 1709-1712. [CrossRef]

32. Ustyantsev, M.A.; Marsal, L.F.; Ferre-Borrull, J.; Pallares, J. Effect of the dielectric background on dispersion characteristics metallo-dielectric photonic crystals. Opt. Commun. 2006, 260, 583-587. [CrossRef] 
33. Qi, L.M.; Yang, Z.Q.; Lan, F.; Gao, X.; Shi, Z.J. Properties of obliquely incident electromagnetic wave in one-dimensional magnetized plasma photonic crystals. Phys. Plasmas 2010, 17, 042501. [CrossRef]

34. Vieu, C.; Carcenac, F.; Pepin, A.; Chen, Y.; Mejias, M.; Lebib, A.; Manin-Ferlazzo, L.; Couraud, L.; Launois, H. Electron beam lithography: Resolution limits and applications. Appl. Surf. Sci. 2000, 164, 111-117. [CrossRef]

35. Liu, N.; Guo, H.C.; Fu, L.W.; Kaiser, S.; Schweizer, H.; Giessen, H. Three-dimensional photonic metamaterials at optical frequencies. Nat. Mater. 2008, 17, 31-37. [CrossRef]

(c) (1)

(C) 2019 by the authors. Licensee MDPI, Basel, Switzerland. This article is an open access article distributed under the terms and conditions of the Creative Commons Attribution (CC BY) license (http://creativecommons.org/licenses/by/4.0/). 